\title{
Return to competition in a chronic low back pain runner: beyond a therapeutic exercise approach, a case report
}

\author{
Sergio Veneziani (1,2), Christian Doria (1), Luca Falciati (3), Claudio Carlo Castelli (2), \\ Giorgio Fanò Illic $(1,4)$ \\ (1) Department of Neurosciences \& Imaging, University “G. D'Annunzio” Chieti-Pescara, \\ Chieti, Italy; (2) Orthopedics \& Traumatology and Emergency\&Urgency Departments, "Papa \\ Giovanni XXIII" Hospital, Bergamo, Italy; (3) Department of Clinical and Experimental \\ Sciences, University of Brescia, Italy; (4) A.S.I. Italian Space Agency, Rome, Italy
}

\begin{abstract}
Chronic low back pain (CLBP) is a disabling condition affecting both quality of life and performance in athletes. Several approaches have been proposed in the field of physiotherapy, manual therapy, physical exercise and counseling. None apparently is outdoing the other with the exception of trunk stability exercises in specific conditions. The present paper describes a clinical success in managing a CLBP runner affected by MRI documented disk herniation via dietary change. Dietary changes allowed our patient that had failed with previous standard therapeutic approaches, to regain an optimal pain-free condition. We advance the hypothesis that a visceral-autonomic concomitant or primary disturbance possibly generating mild gastrointestinal discomfort in CLBP patients should be ruled out as a possible cause of pain and disability at the somato-motor level.
\end{abstract}

Key Words: Low back pain, visceral pain, neural plasticity, central sensitization, irritable bowel syndrome, gluten sensitivity

Eur J Trans Myol - Basic Appl Myol 2014; 24 (3): 203-207

Studies of clinical pain are limited by bias because of cognitive, emotional and social aspects of the matter. Pain is a multidimensional and highly individualized perception that is difficult to quantify and to validate in the clinical setting. As a matter of fact the etiology, natural history, prognostic factors and indicators for chronicity of chronic low back pain (CLBP) remain inconclusive in spite of extensive research effort. Most of these efforts have focused on mechanical and structural aspects of the problem. The structures considered responsible for back pain are classically the intervertebral disk, the facet joints and the highly innervated ligaments in the area.

Such a simplistic biomechanical model of the spinal system has been enhanced by the description of a neuromuscular control unit which helps in stabilizing the spine during various activities of daily living. Muscle itself or more precisely, the neuro-motor unit is a demonstrated source of pain and dysfunction. Specifically, impairments in proprioception may be detected in patients with CLBP when assessed with a motion perception threshold measure. ${ }^{2}$

In a study comparing CLBP patients and healthy subjects, the group receiving motor control exercise showed a greater improvement in transversus abdominis muscle's recruitment compared to the group receiving general exercise or spinal manipulative therapy. ${ }^{3}$ The reasons leading to motor control dysfunctions of the spine are not clear: some authors report that delayed response of trunk muscles could relate to inaccurate information's processing from higher centers of the central nervous system. ${ }^{4}$ Others emphasize a peripheral mechanism mediated by a spinal reflex deficit. ${ }^{5}$

None of the theories are conclusive, thus the present paper advances a brain-gut interaction issue. The braingut interaction, in terms of motor consequences of an inflammatory condition of the digestive tract (and specifically the intestinal mucosa as comorbidity in CLBP), has been proposed back in $2005 .^{6}$ There is also a growing body of evidence on gluten related disorders epidemiology and impact beyond the classical gastrointestinal features for both celiac disease and gluten sensitivity. Both these conditions are likely to be underdiagnosed, as a significant percentage of patients affected by unclassified neuropathic diseases display positive celiac serology in spite of no gastrointestinal symptoms. 


\section{Return to competition in a chronic low back pain runner}

Eur J Trans Myol - Basic Appl Myol 2014; 24 (3): 203-207

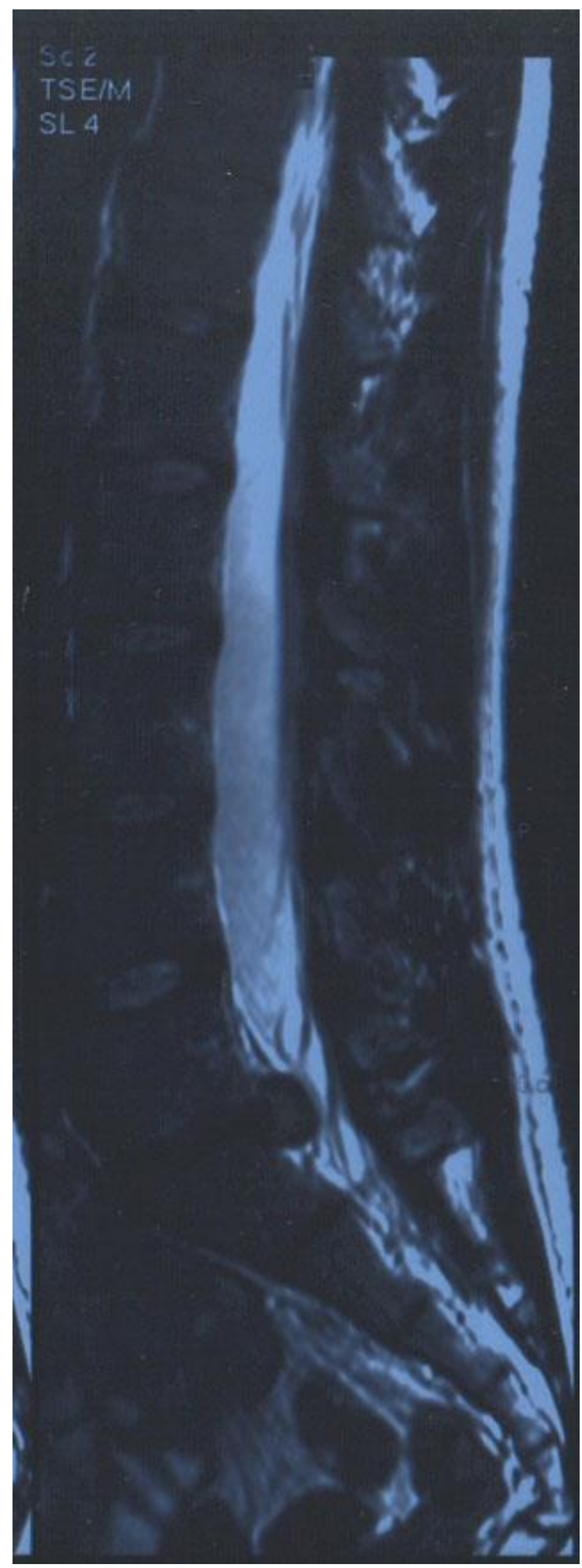

Figure 1 . Lumbosacral spine MRI.

\section{Materials and Methods}

We will discuss a "case report", aware of the advantages and limitations of such a choice. A single case allows one to describe in detail the clinical presentation, therapeutic approach and outcome
Table 1 . List of aliments withdrawn from the daily diet

Gluten

Cow milk

Cow cheese*

Eggs*

Yeast*

Sugar*

Red meat*

Oranges*

Coffe*

Legumes*

* temporarily ( 4 weeks) and indeterminately. An occasional
consumption of the latest has been granted.

evaluation through internationally adopted tools avoiding the loss of data intrinsic to the selection of a representative pool of subjects. On the other hand, even if it is felt that what reported is a common feature of the CLBP population, these observations are intended at stimulating a different perspective on the problem and possibly promote a new direction of investigation. Our subject is a 34 year-old man (1.83 $\mathrm{m}, 71.5 \mathrm{Kg}$ ) reporting a 6 years history of progressive low back pain with sporadic radiation to the left lower limb. His symptoms become noticeably worse during physical workout limiting heavily his activity as an amateur runner of intermediate distances. A lumbosacral spine MRI (2007) showed degenerative changes and disk protrusion with a median-para median herniation compressing the homologous nerve root at the L5-S1 level (Figure 1). He then consulted with a physiatrist that addressed him to the neurosurgeon; the latter excluded neurological deficits indicative of surgery. After a five months course of conservative treatment including specific core stability exercises the clinical picture improved globally so that the subject participated in a competitive $8.5 \mathrm{Kms}$ run with a final time of $38^{\prime} 25^{\prime}$ " and an average running pace of 4'31" $\mathrm{min} / \mathrm{Km}$. Due to persistent debilitating symptomatology he repeated an MRI study in May 2011 that showed the persistence of the herniation with neither a free fragment nor any sign of congestion. He then presented for a consult with the corresponding author. Upon inspection a loss of the physiological lumbar lordosis was noted. On physical exam no major neurologic or ROM deficits were detected. Local pain on palpation at the lumbosacral area and weakness in the rectus femoris and gluteus medium bilaterally were 


\begin{tabular}{ccccc}
\hline & Acute Phase (september 2007) & 2008-2011 & 2011-March 2013 & April 2013 to date \\
\hline VAS & 9,5 & 5 & 7 & 3,5 \\
Roland Morris & Return to compegtition in a chronic low back pain runner 15 & 6 \\
\hline
\end{tabular}

Table 2 . Pain and functional course on the timeline from symptoms presentation to date relatively to significant interventions.

\begin{tabular}{ccccc}
\hline & Acute Phase (september 2007) & 2008-2011 & 2011-March 2013 & April 2013 to date \\
\hline VAS & 9,5 & 5 & 7 & 3,5 \\
Roland Morris & 22 & 8 & 15 & 6 \\
\hline
\end{tabular}

present. Mild tenderness and bloating of the abdomen on palpation were not supported by a positive history of major gastrointestinal disorders. Based on the author experience a restrictive diet (Table 1) was discussed and introduced together with a 20 day course of probiotics, homeopathic and herbal remedies. After the resolution of all symptoms the restrictions were lifted with the exception of gluten and of milk derivatives.

\section{Results and Discussion}

The subject reported a progressive regression of the symptoms as displayed in the scales and questionnaires; (Table 2) these results are maintained to date. There were no confounding factors (besides placebo's effect) that could explain the outstanding clinical result since the inception of the dietary regimen. The subject was instructed not to change his habits in terms of physical activity and did not participate in any additional physical or rehabilitative program. The outcome was not limited to the primary therapeutic goal: as a matter of fact other sources of physical and mental distress improved as evidenced by the SF-36 forms (Figure 2).

The influence of a limited therapeutic intervention at the gastrointestinal level on a variety of apparently unrelated symptoms is not surprising when considering CLBP as comorbidity, hence one finding among the others. In this perspective other subjects might express a more evident symptomatology at the digestive level (irritable bowel syndrome) whether emotional, energy related (chronic fatigue syndrome) or diffused pain muscle pain like in "fibromyalgia". At present, these subjects are managed by professionals with a different background including gastroenterologist, orthopedist, rheumatologist, neurologist, psychiatrist not to mention a slew of individuals under the umbrella of "alternative medicine". CLBP is indeed a condition that challenges the diagnostic and therapeutic capabilities of the professionals involved. To date the rehabilitative and cognitive approaches do not demonstrate fully satisfactory results especially when the treatment plan is not individualized. A systematic review on the effectiveness of physical and rehabilitation interventions for chronic non-specific low back pain showed that unusual approaches like neuromotor oriented patient education (rather than anatomically oriented), acupuncture and yoga provide greater effectiveness on disability than any of the commonly proposed treatments. ${ }^{7}$ CLBP comorbidity with other "vague" diagnoses like chronic fatigue syndrome, anxiety and fibromyalgia and what has been deemed the "functional somatic syndrome" might be overlooked. ${ }^{8}$ Functional gastrointestinal and gluten related disorders are seldom self-reported or under investigation in CLBP patients. Yet the literature clearly associates gut related dysfunction with pain and

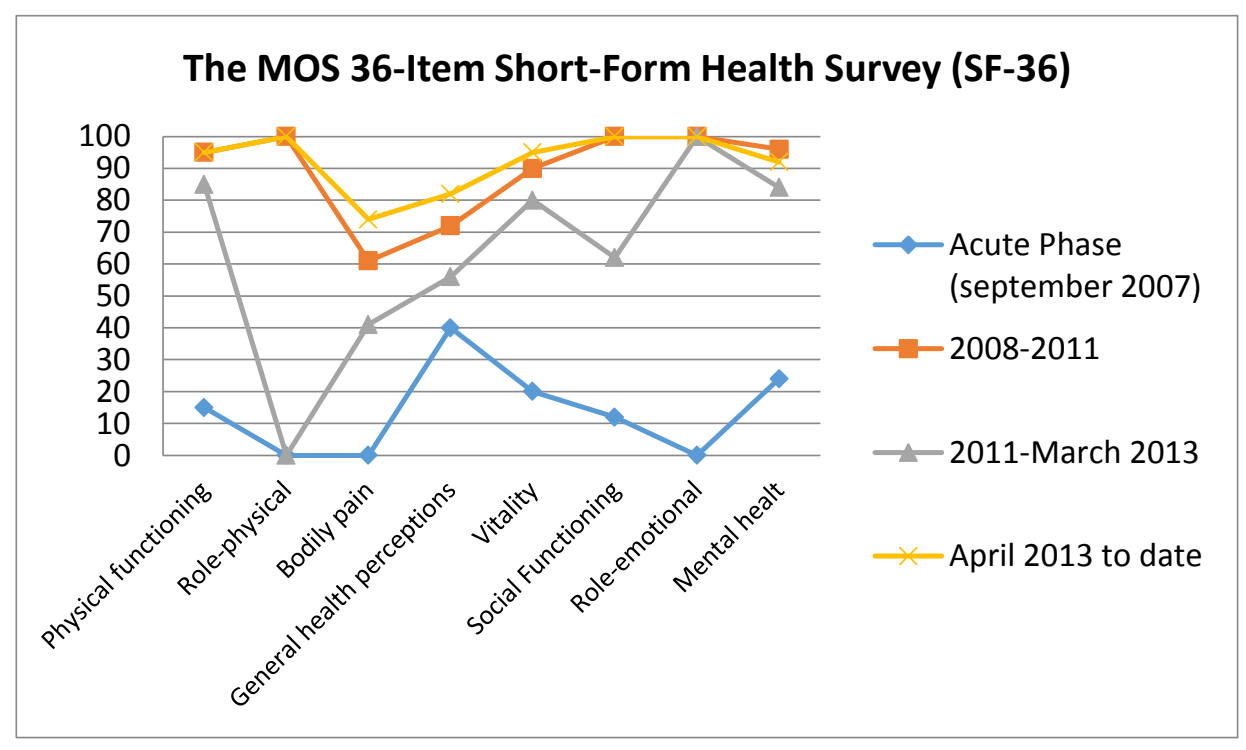

Fig 2. Dietary changes' related impact on a multilevel index of life's quality besides back pain. 


\section{Return to competition in a chronic low back pain runner}

Eur J Trans Myol - Basic Appl Myol 2014; 24 (3): 203-207

sensory-motor abnormalities that in the end could explain spine dysfunction. A vast array of neurological symptoms are related to gluten sensitivity, ${ }^{9,10}$ and gastrointestinal neuromuscular abnormalities observed in animal models seem to justify the symptomatology of gluten sensitive subjects. ${ }^{11}$ In both animal models and humans there is now evidence that chronic visceral pain leads to somatic hypersensitivity and not the opposite as previously described. Specifically in IBS this hypersensitivity is not limited to the colon but displays a vast diffusion and a variety of clinical symptoms representative of alteration in central pain processing. This observation is supported by the discovery of NMDA receptors in the enteric nervous system and their role in central and peripheral sensitization and plasticity, as well as the acknowledged notion of convergence of sensory pathways at the spinal level. ${ }^{12}$ These abnormalities or even only pain in the gut in response to a trigger event, whether perceived or not, are associated with visceral hypersensitivity. This in the end could be the explanation, through plasticity and viscerosomatic convergence of stimuli, of the motor response's alteration as we see in conditions like CLBP. ${ }^{13}$ Neural plasticity is not only the adaptive response of the central nervous system to learning, structural damage or sensory deprivation, but also an increasingly recognized common feature of the gastrointestinal nervous system changes during pathological states. Neural plasticity in the peripheral nervous system and specifically in the enteric nervous system drives neural plasticity in the central nervous system (specifically in the cortex). CLBP as a central cortical disturbance featuring motor neurons activity suppression in the primary motor cortex was recently proposed. ${ }^{14}$ Why this occur has no explanation yet. Visceral stimulation activates the cerebellum and the dorsolateral prefrontal cortex, a critical point of the sensorimotor integrator network. Through connections to the limbic system and cortical associative areas it can affect the integration of motor responses to the stimulus at the cortical level. ${ }^{15}$ Motor training induction of cortical maps reorganization in CLBP brings further evidence of the interconnection of these pathophysiologic events. ${ }^{16}$ Interestingly meridian therapy procedures like acupuncture and acupressure are particularly supported in both IBS (irritable bowel syndrome) and CLBP. Acupuncture modulates both motor and limbic central activity. $^{17,18}$ Two apparently unrelated complaints could be a different manifestations of the same chronic visceral hypersensitivity leading to central plastic changes in sensory motor organization and perception. Hence the efficacy on both symptoms of a technique modulating the serotoninergic pathway. ${ }^{16,18}$ CLBP has been positively associated in cross-sectional studies to cardiovascular disease, headache, respiratory problems, anxiety, depression and IBS. Given that it is a multifaceted issue, it should be considered in terms of comorbidity where the prevalence of other symptoms could be irrelevant or unreported. ${ }^{6}$ The visceral and metabolic aspects of this problem have been overlooked so far as most studies focused on anatomy and abnormal mechanics. Food as a source of inflammation, pain related symptoms and motor impairment should be considered as primarily. Tools in the field of complementary medicine provide a different insight, a chance both for managing the symptoms of a disabling condition and possibly preventing the occurrence of more aggravating disorders. CLBP could be an epiphenomenon of a complex neural plasticity syndrome related to gastrointestinal and/or autonomic dysfunction. More research is needed on this topic indeed, in particular randomized controlled trials.

\section{Acknowledgements}

We thanks Andrea Lanterna, Reparto di Neurochirurgia Azienda Ospedaliera "Papa Giovanni XXIII", Bergamo, Italy and Marcello Caso, Department of Biology, California State University, Sacramento, USA, for their encouragement to write this article.

\section{Contributions of Authors}

SV had the significant role in ideating the experimental design and analyzing the results. Data analysis was also carried out by CD, LF, CCC. GF-I and SV contributed to preparation and critical reading of the paper. The authors declare that they have no conflict of interest.

\section{Corresponding Author}

Sergio Veneziani, Department of Neurosciences \& Imaging, University “G. D’Annunzio” Chieti-Pescara, Chieti, Italy; and Department of Clinical and Experimental Sciences, University of Brescia, Italy. Phone: +390352674260

E-mail: sergio@veneziani.biz

\section{References}

1. Panjabi M. The stabilizing system of the spine. Part I. Function, dysfunction, adaptation, and enhancement. J Spinal Disord 1992;5:383-9.

2. Lee AS, Cholewicki J, Reeves NP, et al. Comparison of trunk proprioception between patients with low back pain and healthy controls. Arch Phys Med Rehabil 2010;91:1327-31.

3. Ferreira PH, Ferreira ML, Maher CG, et al. Changes in recruitment of transversus abdominis muscle correlate with disability in people with chronic low back pain. $\mathrm{Br} \mathrm{J}$ Sports Med 2010;44:1166-72.

4. Leinonen V, Airaksinen M, Taimela S, et al. Low back pain suppresses preparatory and triggered upper-limb activation after sudden upper-limb loading. Spine 2007;32:E150-5. 


\section{Return to competition in a chronic low back pain runner}

Eur J Trans Myol - Basic Appl Myol 2014; 24 (3): 203-207

5. Reeves NP, Cholewicki J, Milner TE. Muscle reflex classification of low-back pain. J Electromyogr Kinesiol 2005;15:53-60.

6. Holmberg S, Thelin A, Stiernstrom E, Svardsudd $\mathrm{K}$. Low back pain comorbidity among male farmers and rural referents: a population-based study. Ann Agric Environ Med 2005;12(2):261-8.

7. van Middelkoop M, Rubinstein S M, Kuijpers T, et al. A systematic review on the effectiveness of physical and rehabilitation interventions for chronic non-specific low back pain. Eur Spine J 2011;20:19-39.

8. Masuko K, Nakamura H. Functional somatic syndrome: how it could be relevant to rheumatologists. Mod Rheumatol 2007;17:17984.

9. Hadjivassiliou M, Sanders DS, Grünewald RA, et al. Gluten sensitivity: from gut to brain. Lancet Neurol 2010;9:318-30. doi: 10.1016/S14744422(09)70290-X.

10. Kozanoglu E, Basaran S, Goncu MK. Proximal myopathy as an unusual presenting feature of celiac disease. Clin Rheumatol 2005;24.1:76-8.

11. Volta U, De Giorgio R. New understanding of gluten sensitivity. Nat Rev Gastroenterol Hepatol2012;9:295-9.

12. Zhou Q, Verne GN. NMDA Receptors and Colitis: Basic Science and Clinical Implications. Reviews in analgesia 2008;10:33.
13. Azpiroz F, Dapoigny M, Pace F, et al. Nongastrointestinal disorders in the irritable bowel syndrome. Digestion 2000;62:66-72.

14. Aziz Q, Thompson DG, Ng VWK, et al. Cortical processing of human somatic and visceral sensation. J Neurosci 2000;20:2657-63.

15. Tsao H, Galea MP, Hodges PW. Driving plasticity in the motor cortex in recurrent low back pain. Eur J Pain 2010;14:832-9. doi: 10.1016/j.ejpain.2010.01.001. Epub 2010 Feb 23.

16. Tian XY, Bian ZX, Hu XG, et al. Electroacupuncture attenuates stress-induced defecation in rats with chronic visceral hypersensitivity via serotonergic pathway. Brain Res 2006;1088:1018. Epub 2006 May 2.

17. Maioli C, Falciati L, Marangon M, Perini S, Losio A. Short- and long-term modulation of upper limb motor-evoked potentials induced by acupuncture. Eur J Neurosci 2006;23:1931-8.

18. Zhang WB, Wu A, Litscher G, Chae Y. Effects and mechanism of acupuncture based on the principle of meridians. Evid Based Complement Alternat Med 2013;2013:684027. doi: 10.1155/2013/684027. Epub 2013 Nov 27.

19. Maes M, Coucke F, Leunis JC. Normalization of the increased translocation of endotoxin from gram negative enterobacteria (leaky gut) is accompanied by a remission of chronic fatigue syndrome. Neuro Endocrinol Let 2007;28:739-44. 